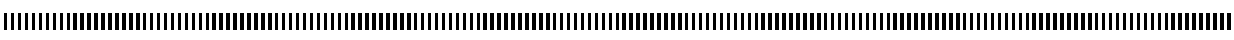

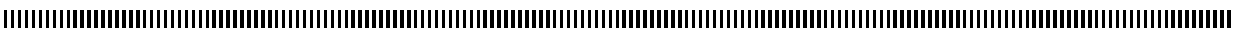

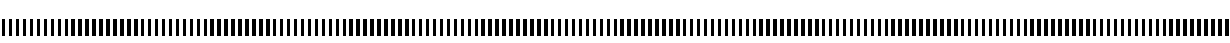

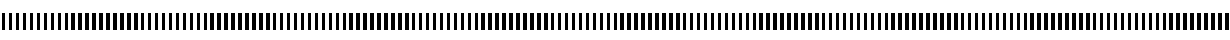

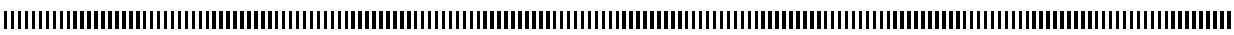

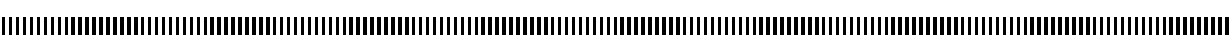

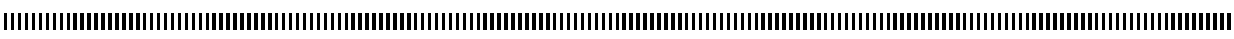

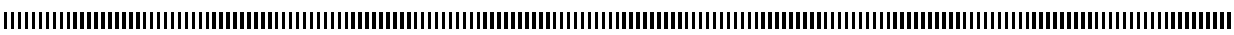

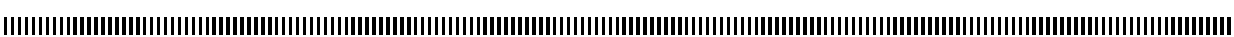

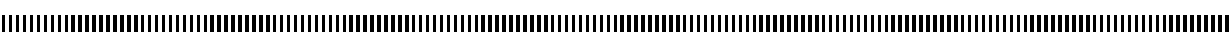

\title{
Spectral Procedure with Diagonalization of Operators for 2D Navier-Stokes and Heat Equations in Cylindrical Geometry
}

\author{
El Mahdi El Guarmah*, Abdelkhalek Cheddadi*, Mejdi Azaiez ** \\ * Modelization and Scientific Computing, Mohammadia Engineering School, Mohamed V Univer- \\ sity, Ibn Sina Str., POB 765 Agdal, Rabat, Morocco \\ guarmah@hotmail.com; cheddadi@emi.ac.ma \\ ** Université Bordeaux 1, TREFLE UMR 8505, 33607 Pessac, France \\ azaiez@enscpb.fr
}

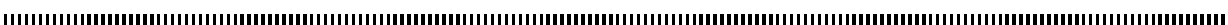

ABSTRACT. We present in this paper a spectral method for solving a problem governed by NavierStokes and heat equations. The Fourier-Chebyshev technique in the azimuthal direction leads to a system of Helmholtz equations. The Collocation-Chebyshev method in the radial direction has been used for the simulation of these equations. The Crank-Nicholson scheme is employed to solve the Helmholtz systems obtained for wide ranges of parameters, and its efficiency is considerably improved by diagonalization of the obtained operators. The results are in a very good agreement with the experimental data available in the literature.

RÉSUMÉ. Dans ce papier, une méthode spectrale est appliquée à un problème gouverné par les équations de Navier-Stokes couplées à celle de la chaleur pour un fluide visqueux incompressible à nombre de Prandtl fini, confiné dans un espace annulaire. indent La technique de Fourier-Chebyshev dans la direction azimutale est mise en oeuvre et mène à un système d'équations couplées du type Helmholtz. La méthode de Collocation-Chebyshev dans la direction radiale a été employée pour la simulation de ces équations. Le schéma de Crank-Nicholson est implémenté pour résoudre les équations de Helmholtz obtenues pour une large gamme de paramètres, et l'efficacité de la procédure de calcul est considérablement améliorée par diagonalisation des opérateurs obtenus. Les résultats sont dans un très bon accord avec les données expérimentales disponibles dans la littérature.

KEYWORDS : Navier-Stokes, Heat equation, Chebyshev Gauss-Lobatto, Crank-Nicholson, Diagonalization, Spectral Space, Physical Space

MOTS-CLÉS : Navier-Stokes, Equation de la chaleur, Gauss-Lobatto Chebyshev, Crank-Nicholson, Diagonalisation, Espace Spectral, Espace Physique

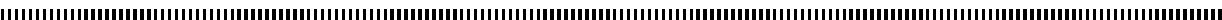

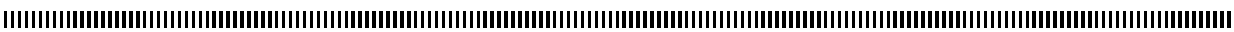

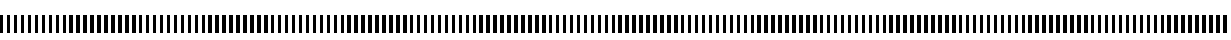

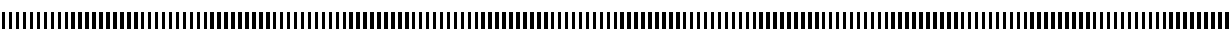
| | |

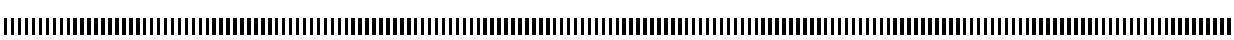
| НИЕ 


\section{Introduction}

We present in this work, a numerical study of an incompressible fluid, in laminar flow between two horizontal concentric coaxial cylinders, with inner and outer radii $r_{i}$ and $r_{e}$, respectively (see Figure 1). The inner cylinder is considered to be held at a uniform temperature $T_{i}$, and the outer cylinder at a uniform temperature, $T_{e}$ with $T_{i}>T_{e}$.

This problem currently receives considerable attention due to its numerous technological practical applications. A very thorough literature survey and comprehensive analysis have been made on the concentric annuli by Kuehn and Goldstein $[8,9]$. They have conducted both numerical simulation using finite difference method of relaxation type, and experimental study using Mach-Zehnder interferometer. Application of other type of finite difference method, using an Alternating-Direction Implicit (ADI) procedure in connection with Artificial Compressibility, has also been reported by Cheddadi and al $[3,4]$ in solving the laminar flow in horizontal concentric annuli, formulated in polar coordinates.

In the present paper, to extend the existing knowledge on the numerical simulation of natural convection heat transfer in horizontal cylindrical annuli, numerical analysis has been made using spectral method based on Collocation-Chebyshev. Prandtl number is fixed at that of air, $\operatorname{Pr}=0.7$.

The present paper contains five sections. The second section will be dedicated to the description of the physical problem and the presentation of the mathematical model. Using a conformal transformation, the governing equations are transformed to allow the use of a pseudo-spectral method. Then, we present a resolution method which allows us to obtain a new system constituted by Helmholtz equations.

We propose then the Collocation Chebyshev method to solve the problem described by the algebraic systems of Helmholtz equations, that we resolve by explicit and implicit iterative methods, in the context of diagonalization of the operators.

We present, finally, the numerical results, for various values of Rayleigh number, and radius ratio. 


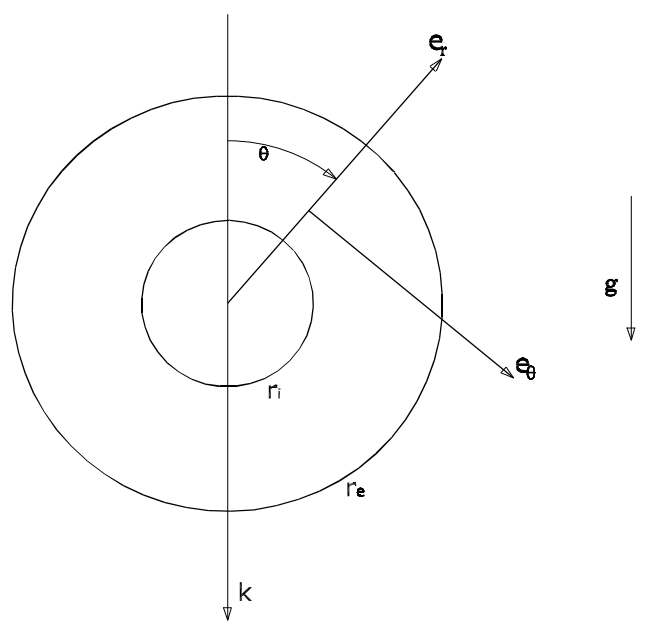

Figure 1 : Definition scheme

\section{Governing equations}

\subsection{General equations of convection}

The problem under consideration is that of two-dimensional laminar convection in an annular geometry. The fluid flow is assumed to be laminar, incompressible Newtonian with constant physical properties and governed by equations of :

Mass conservation principle :

The equation of mass conservation, known as the continuity equation, is :

$$
\operatorname{div} \mathbf{V}=0
$$

where $\mathbf{V}$ is the velocity vector.

Momentum conservation principle ( Navier-Stokes) :

The momentum equation is :

$$
\rho\left(\frac{\partial \mathbf{V}}{\partial t}+\mathbf{V} \cdot \operatorname{grad} \mathbf{V}\right)=-\operatorname{grad} p+\rho \mathbf{g}+\mu \Delta \mathbf{V}
$$

where $p$ is the pressure, $\rho$ the density, $\mu$ the dynamic viscosity, $\mathbf{g}$ the gravitational acceleration vector $\mathbf{g}=\mathbf{g k}, \mathbf{k}$ the vertical unitarian vector descendant. 


\section{Energy conservation principle :}

The equation of energy conservation takes the form :

$$
\frac{\partial T}{\partial t}+\mathrm{V} \cdot \operatorname{grad} T=\kappa \Delta T
$$

where $\kappa$ is the thermal diffusivity.

\section{Boundary conditions}

All the boundaries are considered impermeable and isothermal, the boundary conditions are given as :

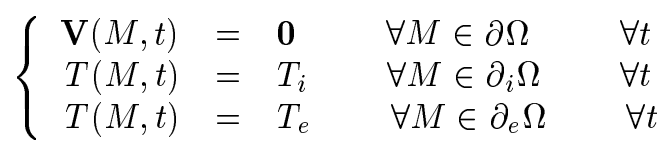

with $\partial i \Omega$ and $\partial e \Omega$ the inner and outer limit surfaces, respectively, and $\partial \Omega=\partial i \Omega \cup \partial e \Omega$.

We suppose that the geometry is infinite in the $z$ direction, so that the problem could be considered as two-dimensional.

\subsection{Simplifying hypotheses}

These equations are generally simplified under the following hypotheses :

\section{- State equation of the fluid}

The fluid flow is assumed to be incompressible, the density is linearized as :

$$
\rho=\rho_{0}\left[1-\beta\left(T-T_{e}\right)\right]
$$

with $\rho_{0}$ is the reference density, and $\beta$ is the coefficient for thermal expansion.

\section{- Boussinesq approximation}

In natural convection, the Boussinesq approximation simplifies the Navier-Stokes equations. The density of the fluid is assumed to be constant except in the natural convection term $(\rho \mathbf{g})$. An extensive study of validity of this approximation is presented in [6]. 


\subsection{Dimensionless equations}

The following nondimensional quantities are introduced :

$$
\left\{\begin{aligned}
(x, y)_{+}=\frac{(x, y)}{r_{i}} & t_{+}=\frac{\kappa t}{r_{i}^{2}} \quad p_{+}=\frac{r_{i}^{2}\left(p+\rho_{0} g \mathbf{z}\right)}{\rho_{0} \kappa^{2}} \\
V_{+}=\frac{V}{\kappa / r_{i}} & T_{+}=\frac{T-T_{e}}{T_{i}-T_{e}}
\end{aligned}\right.
$$

The dimensionless steady-state governing equations (1,2 and 3 ) can then be written (where indication " + " is omitted for all the non-dimensional variables), as follows :

$$
\left\{\begin{aligned}
\operatorname{div} \mathbf{V} & =0 \\
\frac{\partial \mathbf{V}}{\partial t}+\mathbf{V} \cdot \operatorname{grad} \mathbf{V} & =-\operatorname{grad} p-\operatorname{Ra} \operatorname{Pr} T \mathbf{k}+\operatorname{Pr} \Delta \mathbf{V} \\
\frac{\partial T}{\partial t}+\mathbf{V} \cdot \operatorname{grad} T & =\Delta T
\end{aligned}\right.
$$

where $R a=\frac{\rho_{0} g \beta r_{i}^{3}\left(T_{i}-T_{e}\right)}{\mu \kappa}$ and $\operatorname{Pr}=\frac{\mu}{\rho_{0} \kappa}$ are the Rayleigh and Prandtl numbers, respectively. A second Rayleigh number $R a l=R a(R-1)^{3}$, where $R$ is the radius ratio $R=\frac{r_{e}}{r_{i}}$, is introduced and will serve for presenting the results, to allow comparison with the Rayleigh-Benard problem.

The boundary conditions become :

$$
\left\{\begin{array}{lccc}
\mathbf{V}(M, t)=\mathbf{0} & \forall M \in \partial \Omega & \forall t \\
T(M, t)=1 & \forall M \in \partial_{i} \Omega & \forall t \\
T(M, t)=0 & \forall M \in \partial_{e} \Omega & \forall t
\end{array}\right.
$$

\subsection{Vorticity-stream function formulation}

In order to eliminate pressure, the governing equations are expressed in terms of the vorticity, $\omega$, and stream-function, $\Psi$. Using the polar coordinates, the stream-function is related to the velocity $\mathbf{V}=u \overrightarrow{e_{r}}+v \overrightarrow{e_{\theta}}$ by:

$$
\left\{\begin{array}{l}
u=\frac{1}{r} \frac{\partial \Psi}{\partial \theta} \\
v=-\frac{\partial \Psi}{\partial r}
\end{array}\right.
$$

and the vorticity is given by : $\omega=-\left(\frac{\partial^{2} \Psi}{\partial r^{2}}+\frac{1}{r} \frac{\partial \Psi}{\partial r}+\frac{1}{r^{2}} \frac{\partial^{2} \Psi}{\partial \theta^{2}}\right)$ 
The governing system (7) of the dimensionless stream-function, vorticity and temperature in the polar coordinate system $(r, \theta)$ is as follows :

$$
\left\{\begin{array}{l}
\Delta \Psi=-\omega \\
\Delta \omega=\operatorname{Ra}\left(\frac{\cos \theta}{r} \frac{\partial T}{\partial \theta}+\sin \theta \frac{\partial T}{\partial r}\right)+\frac{1}{r P r}\left(\frac{\partial \Psi}{\partial \theta} \cdot \frac{\partial \omega}{\partial r}-\frac{\partial \Psi}{\partial r} \cdot \frac{\partial \omega}{\partial \theta}\right) \\
\Delta T=\frac{1}{r}\left(\frac{\partial \Psi}{\partial \theta} \frac{\partial T}{\partial r}-\frac{\partial \Psi}{\partial r} \frac{\partial T}{\partial \theta}\right)
\end{array}\right.
$$

The boundary conditions accompanying (10) are :

$$
\left\{\begin{array}{rrrrrrrr}
r= & : & \Psi=0 & , & \frac{\partial \Psi}{\partial r}=0 & , & T=1 & \forall \theta \\
r= & : \quad & \Psi=0 &
\end{array}\right.
$$

where $R$ is the radius ratio : $R=\frac{r_{e}}{r_{i}}$.

Because of the existence of a vertical symmetry plane in the considered geometry, the problem is solved only for the vertical half plane. The stream-function is constant along each wall, as well as along the lines of symmetry.

$$
\left\{\begin{array}{llll}
\theta=0 \text { and } \theta=\pi \quad: \quad & \Psi=0 \quad \text { and } \quad \frac{\partial T}{\partial \theta}=0 \quad \forall r \\
\theta=0 \text { and } \theta=\pi \quad: \quad \omega=0 &
\end{array}\right.
$$

\section{Numerical analysis}

\subsection{Problem formulation}

Interval $[1, R]$ can be mapped to $[0, \ln R]$, and then to $[-1,+1]$, so that Chebyshev polynomials could be applied. We operate the transformation : $X=\ln r, Y=\theta$ (see Figure 2), so that the initial domain $[1, R] \mathrm{x}[0, \pi]$ is transformed in a cartesian domain 
$[0, \ln R] \times[0, \pi]$

$\omega=0$

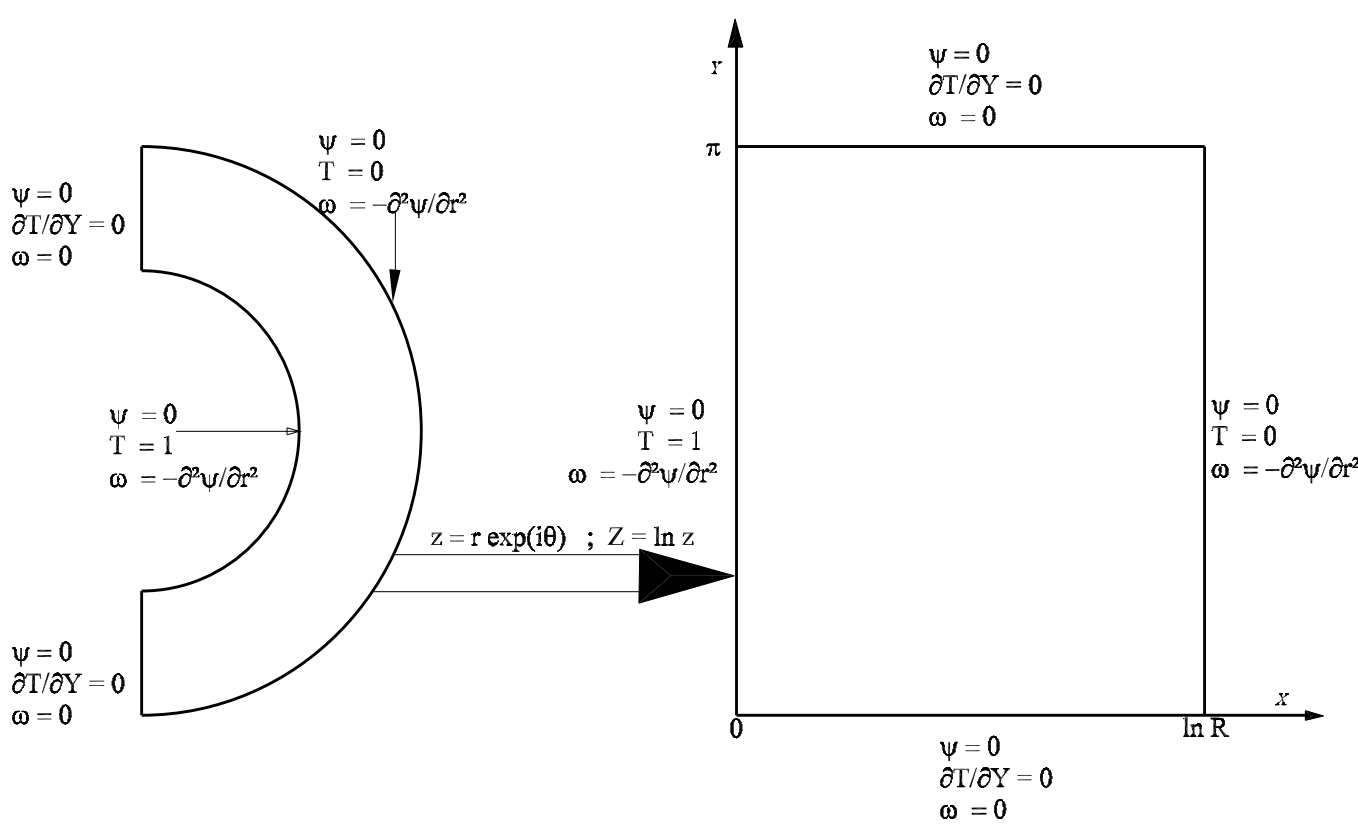

Figure 2. Conformal transformation

\subsection{Chebyschev approximation}

We adopt Fourier-Chebyshev approximation in the azimuthal direction and the CollocationChebyshev approximation in the radial direction $[5,10]$. The general Fourier Series representation of the solutions $\Psi, \omega$ and $T$ for the order $N$, write as follows :

$$
\left\{\begin{aligned}
\Psi_{N} & =\sum_{p=0}^{N} f_{p}(X) \sin (p y) \\
\omega_{N} & =\sum_{p=0}^{N} h_{p}(X) \sin (p y) \\
T_{N} & =1-\alpha X+\sum_{p=0}^{N} g_{p}(X) \cos (p y)
\end{aligned}\right.
$$

where $\alpha$ is a characteristic parameter of the geometry $\left(\alpha=\frac{1}{\ln R}\right)$, and the function $(1-$ $\alpha X)$ is a particular solution allowing the caption of the boundary conditions for $T$. So that Chebyshev polynomials could be applied, we set $x=2 \alpha X-1$ where $x \in[-1,1]$. 
System (13) is substituted into the dimensional governing $\Psi-\omega-T$ equations (10). After reorganizing the terms, the dimensionless governing equations result in the hereafter coupled non-linear Helmholtz equations. The Helmholtz equations can be written :

For $1 \leq p \leq N$

$\left\{\begin{aligned} f_{p}^{\prime \prime}(x)-\frac{p^{2}}{4 \alpha^{2}} f_{p}(x) & =F_{p}(x) \\ h_{p}^{\prime \prime}(x)-\frac{p^{2}}{4 \alpha^{2}} h_{p}(x) & =H_{p}(x) \\ g_{p}^{\prime \prime}(x)-\frac{p^{2}}{4 \alpha^{2}} g_{p}(x) & =G_{p}(x)\end{aligned}\right.$

for $p=0$ :

$$
g_{0}^{\prime \prime}(x)=G_{0}(x)
$$

where $x=2 \alpha X-1, x \in[-1,1]$.

The boundary conditions become :

$$
\left\{\begin{array}{rlrlrl}
f_{p}(x=-1) & =0 & f_{p}(x=1) & =0 \\
f_{p}{ }^{\prime}(x=-1) & =0 & f_{p}^{\prime}(x=1) & =0 \\
g_{p}(x=-1) & =0 & g_{p}(x=1) & =0 \\
h_{p}(x=-1) & =-4 \alpha^{2} f_{p}{ }^{\prime \prime}(x=-1) & h_{p}(x=1) & & =-4 \alpha^{2} e^{-\frac{2}{\alpha}} f_{p}{ }^{\prime \prime}(x=1)
\end{array}\right.
$$

with

$$
\begin{aligned}
& \left\{\begin{aligned}
F_{p}(x) & =-\frac{1}{4 \alpha^{2}} \exp \left(\frac{x+1}{\alpha}\right) h_{p} \\
H_{p}(x) & =\frac{1}{4 \alpha \operatorname{Pr}}\left[\sum_{j=1}^{N}\left[h^{\prime}{ }_{j}\left((p-j) f_{p-j}+(j-p) f_{j-p}-(p+j) f_{p+j}\right)\right]\right. \\
& \left.-j h_{j}\left(f^{\prime}{ }_{p+j}+f^{\prime}{ }_{p-j}-f^{\prime}{ }_{j-p}\right)\right] \\
+ & \frac{\operatorname{Raexp}\left(\frac{x+1}{2 \alpha}\right)}{8 \alpha^{2}}\left[2 \alpha \delta g^{\prime}{ }_{p-1}-(p-1) g_{p-1}-2 \alpha \delta_{1 p}-2 \alpha g_{p+1}^{\prime}-(p+1) g_{p+1}\right] \\
G_{p}(x) & =\frac{1}{4 \alpha}\left[-p f_{p}+\sum_{j=0}^{N}\left[j g_{j}\left(f^{\prime}{ }_{j+p}+f_{j-p}^{\prime}-f_{p-j}^{\prime}\right)\right]\right. \\
& \left.\quad+g^{\prime}{ }_{j}\left((p+j) f_{p+j}+(p-j) f_{p-j}+(j-p) f_{j-p}\right)\right]
\end{aligned}\right. \\
& \text { for } p=0 \text { : } \\
& G_{0}(x)=\frac{1}{4 \alpha} \sum_{j=1}^{N}\left(j g_{j}{f^{\prime}}_{j}+j f_{j} g^{\prime}{ }_{j}\right)
\end{aligned}
$$

where

$$
\left\{\begin{array}{llll}
\delta=2 & \delta_{1 p}=1 & \text { for } & p=1 \\
\delta=1 & \delta_{1 p}=0 & \text { for } & p>1
\end{array}\right.
$$


The system (14) is discretized using the collocation - Chebyshev method [2]. The problem can be solved in the spectral space or in the physical space. In the present work, all calculations which we are going to make by means of Collocation-Chebyshev method, were made in the physical space.

The transform matrices $[P S]$ and $[S P]$ between the physical space and the spectral space are used $[1,2]$, in order to study the convergence in the spectral space.

When the Collocation-Chebyshev method is employed, every function is associated with its vector formed by the values of the function at the $(M+1)$ collocation points defined by $x_{k}=\cos \left(\frac{k \pi}{M}\right)$ for $0 \leq k \leq M$. These are the nodes of the Gauss-Lobatto quadrature formula relative to the Chebyshev weight $w(x)=\frac{1}{\sqrt{1-x^{2}}}$.

Second derivative functions $\left(f_{p}{ }^{\prime \prime}, g_{p}{ }^{\prime \prime}, h_{p}{ }^{\prime \prime}\right)$ are calculated from $f_{p}, g_{p}$ and $h_{p}$ respectively by applying a product matrix $\mathrm{x}$ vector, such as $\overrightarrow{f_{p}^{\prime \prime}}=D^{2} \overrightarrow{f_{p}}$, where $D$ is a derivative not symmetric matrix [2]. Spectral Collocation approximation leads to a linear system : for $1 \leq p \leq N$

$$
\left\{\begin{aligned}
D^{2} \vec{f}_{p}-\frac{p^{2}}{4 \alpha^{2}} \vec{f}_{p} & =\vec{F}_{p} \\
D^{2} \overrightarrow{g_{p}}-\frac{p^{2}}{4 \alpha^{2}} \overrightarrow{g_{p}} & =\vec{G}_{p} \\
D^{2} \overrightarrow{h_{p}}-\frac{p^{2}}{4 \alpha^{2}} \overrightarrow{h_{p}} & =\vec{H}_{p}
\end{aligned}\right.
$$

for $p=0$ we have :

$$
D^{2} \overrightarrow{g_{0}}=\overrightarrow{G_{0}}
$$

\subsection{Diagonalization}

The derivative matrices $D_{C L}^{2}$ are obtained by taking into account the boundary conditions. The new matrices are diagonalizabe with simple negative eigenvalues. Algebraic system (20) is then transformed by performing a diagonalization of the operators as introduced in [7]. The system (20) can be written in the eigenspace using Crank-Nicholson scheme as follows :

$$
\left[f_{i}\right]^{n+1}=\frac{\left(1+\frac{\Delta t}{2} \lambda_{i}-\frac{\Delta t}{2} \frac{p^{2}}{4 \alpha^{2}}\right)\left[f_{i}\right]^{n}-\Delta t\left[F_{i}\right]^{n}}{\left(1-\frac{\Delta t}{2} \lambda_{i}+\frac{\Delta t}{2} \frac{p^{2}}{4 \alpha^{2}}\right)}
$$

where $\left[f_{i}\right]$ represents the vector $\overrightarrow{f_{p}}$ (respectively $\overrightarrow{g_{p}}, \overrightarrow{h_{p}}$ ) in the basis of eigenvectors, $\left[F_{i}\right]$ represents the vector $\vec{F}_{p}$ (respectively $\overrightarrow{G_{p}}, \overrightarrow{H_{p}}$ ) in the same basis and $\lambda_{i}$ represents the $i^{t h}$ 
eigenvalue. The exponents $n$ and $n+1$ indicate successive moments. We return in the physical space by multiplying all the vectors by the corresponding passage matrices.

\section{Results and Discussions}

In the present study the Prandtl number was kept fixed at $\operatorname{Pr}=0.7$. All of the results are described only on a semi annular region since the problem is symmetric.

The numerical code is written in $C^{++}$. Numerical solutions are obtained for cylinders with a radius ratio $1.2 \leq R \leq 2.7$.

The results obtained are presented graphically in the form of streamline and isotherm contour plots (see Figures 3 and 4). The heat transfer characteristics, velocity and temperature profiles (see Figure 5 and 6) are studied.

The results of the present study were found valid when compared with experimental and numerical investigations carried out in the past.

Tables 1,2 give the $\Psi_{\max }$ and the global Nusselt number $N u g$ variations, the latter characterizes the heat transfer in the cavity and is given by :

$$
N u_{g}=-\frac{1}{\pi} \ln R \int_{0}^{\pi}\left(\frac{\partial T}{\partial r}\right)_{r=1} d \theta,
$$

according to Ral, $M$ and $N$ with $R=1.6$ and $R=2$ for Crank-Nicholson scheme.

The validation of the code by the explicit method does not constitute a problem as long as $R a l \leq 3000$ and $M \leq 14$. To enhance our code, Crank-Nicholson semi-implicit method is used to avoid instabilities, allowing thus to obtain reliable results for Ral $>3000$ and $M>14$.

\begin{tabular}{|l|l|l|l|l|l|l|}
\hline Ral & 2000 & 3000 & 5000 & 5000 & 5000 & 10000 \\
\hline$M$ & 14 & 30 & 30 & 45 & 50 & 50 \\
\hline$N$ & 30 & 20 & 30 & 45 & 50 & 50 \\
\hline$N u g$ & 1.093 & 1.179 & 1.347 & 1.347 & 1.347 & 1.655 \\
\hline$\Psi_{\max }$ & 4.842 & 7.104 & 10.922 & 10.919 & 10.929 & 18.022 \\
\hline
\end{tabular}

Table 1 Variation of $\psi_{\max }$ and $N u g$ according to $R a l, M$ and $N$ for $R=1.6$ and $\operatorname{Pr}=0.7$ : Crank-Nicholson scheme.

\begin{tabular}{|l|l|l|l|l|}
\hline Ral & 2000 & 3000 & 5000 & 10000 \\
\hline$M$ & 20 & 30 & 50 & 50 \\
\hline$N$ & 20 & 30 & 50 & 50 \\
\hline$N u g$ & 1.165 & 1.290 & 1.499 & 1.837 \\
\hline$\Psi_{\max }$ & 4.650 & 6.597 & 9.782 & 15.234 \\
\hline
\end{tabular}

Table 2 Variation of $\psi_{\max }$ and $N u g$ according to $R a l, M$ and $N$ for $R=2$ and $\operatorname{Pr}=0.7$ : Crank-Nicholson scheme. 

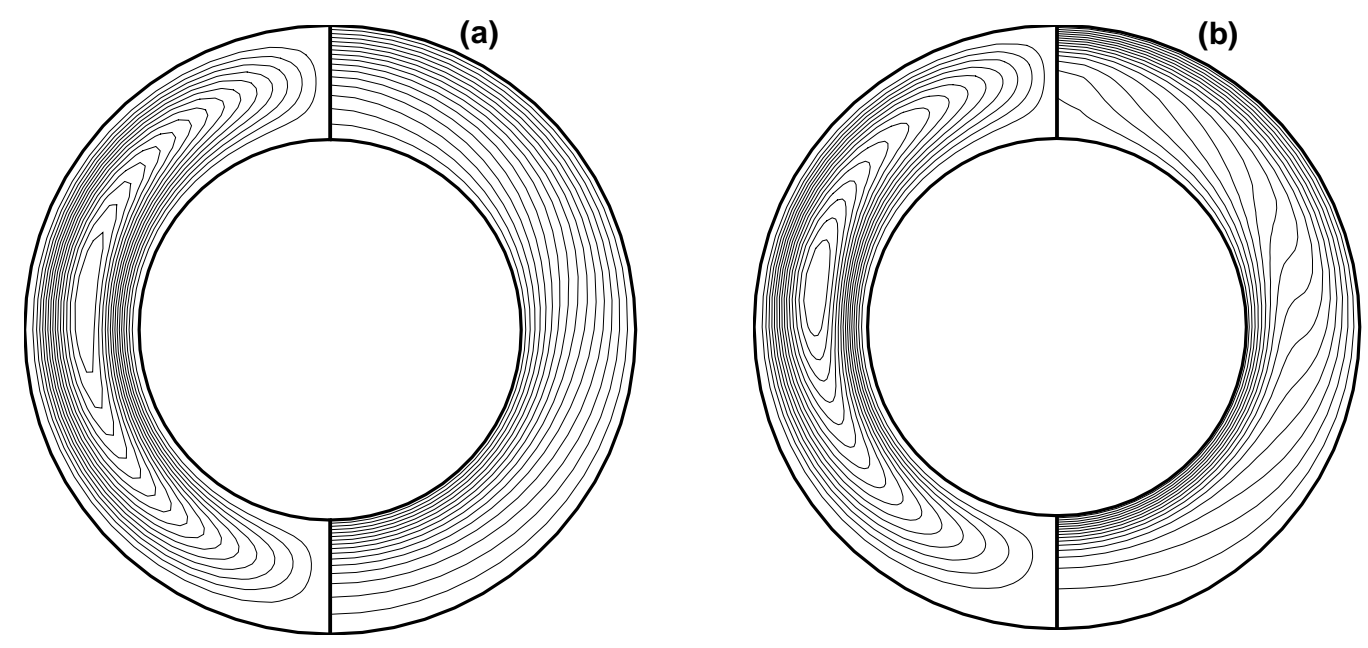

Figure 3 : Isotherms and streamlines for $\mathrm{R}=1.6$

(a) Ral $=3000, \mathrm{M}=30$ and $\mathrm{N}=30$

(b) Ral $=10000, \mathrm{M}=50$ and $\mathrm{N}=50$
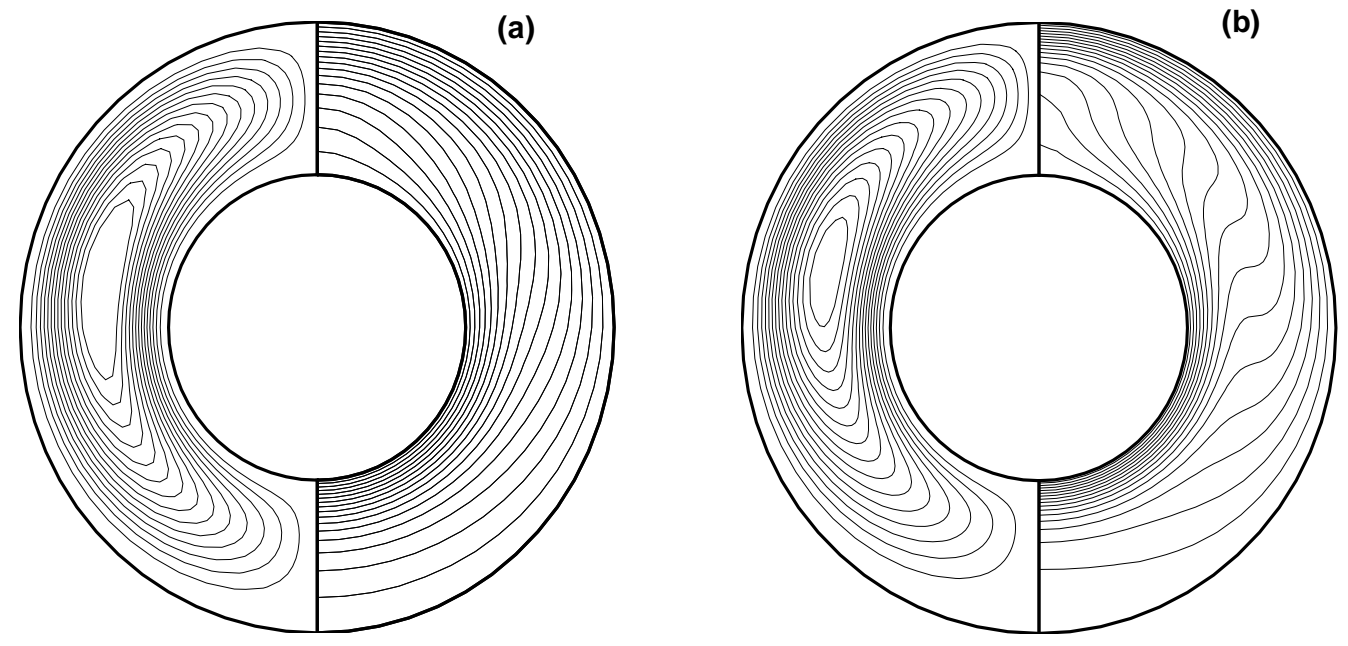

Figure 4 : Isotherms and streamlines for $\mathrm{R}=2$

(a) Ral=3000, M=30 and $\mathrm{N}=30$

(b) Ral $=10000, \mathrm{M}=50$ and $\mathrm{N}=50$ 
Figures 3 and 4 show typical results obtained for annuli with Rayleigh number $R a l=$ 3000 and $R a l=10000$, radius ratio $R=1.6, R=2$ and for different values of $M$ and $N$. As mentioned earlier, the problem under consideration is symmetrical with respect to a vertical axis, and it is found advantageous to reproduce computed results on a single graph with the isotherms on the right half of the cavity and flow pattern on the left half. The fluid near the outer cylinder is heavier and is moving downward while the relatively lighter fluid near the inner cylinder is moving upward. As a consequence of the symmetry and the continuity, the resulting fluid motion inside the whole cavity consists of two counter rotating vortices. Figures 3 and 4 show only the left counter vortex.

The distortion of isotherms in Figures 3 (b) and 4 (b) reveals a strong convective motion inside the cavity. It is also noticed that the maximum heat transfer, indicated by closely spaced isotherms, is located at the top of the cavity for the outer cylinder and at the bottom for the inner one.

Figure 5 shows $T$ versus $r$ for the case with $R a l=10000$ and $R=1.6$ and Figure 6 shows $\Psi$ versus $r$ for the case with $R a l=3000$ and $R=1.6$. As already mentioned, this case corresponds to the presence of single counter circulations in the left half and in the right half cavity. As Ral increases, it is seen that the temperature gradient in the boundary layer adjacent to the outer cylinder increases considerably as the angular position decreases from $T=28 \pi / 30$ towards $T=2 \pi / 30$.

Figure 7 shows the spectral convergence by representing decimal logarithm of spectra coefficients according to $j$.

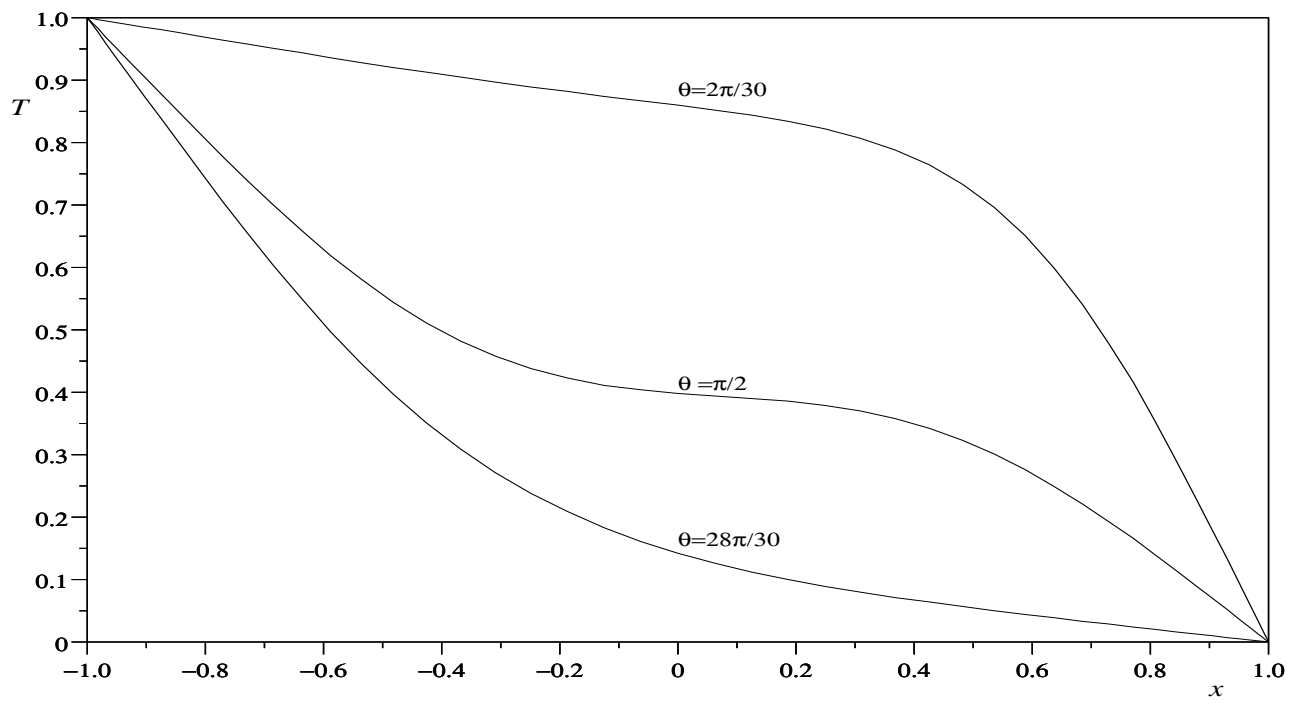

Figure 5 : Temperature profile for $\mathrm{R}=1.6, \mathrm{Ral}=10000, \mathrm{M}=50$ and $\mathrm{N}=50$ 


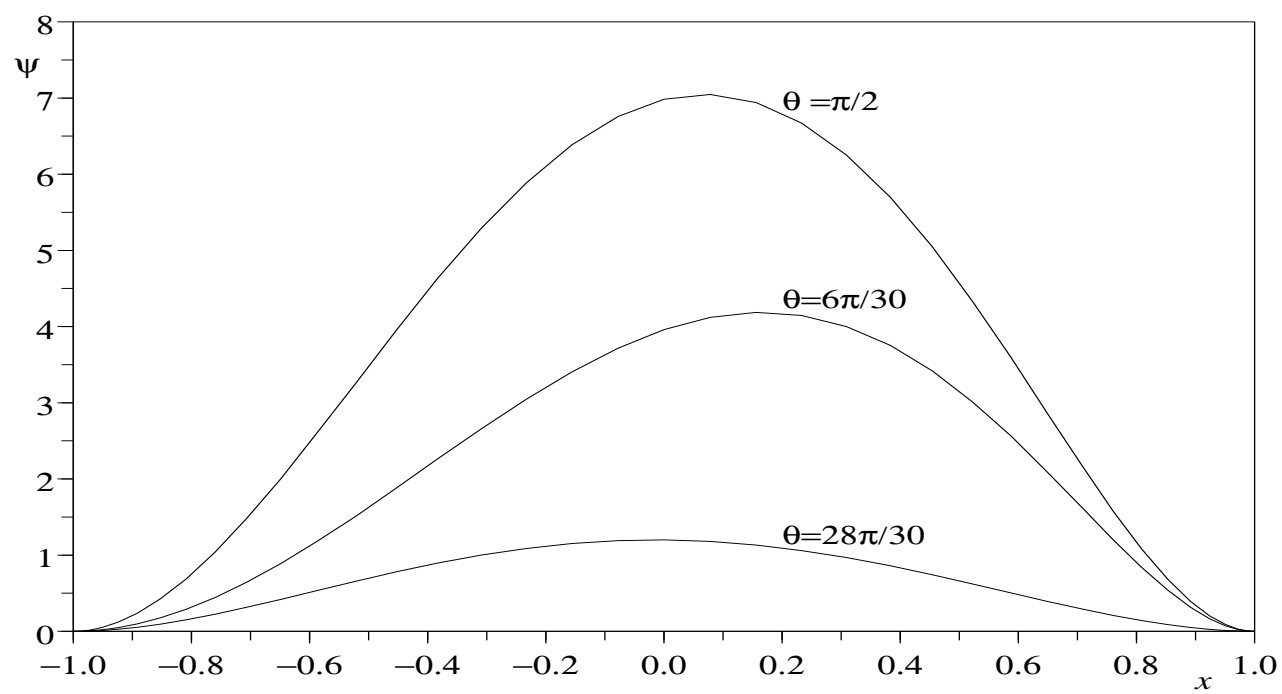

Figure 6 : Stream function profile for $\mathrm{R}=1.6, \mathrm{Ral}=3000, \mathrm{M}=40$ and $\mathrm{N}=30$

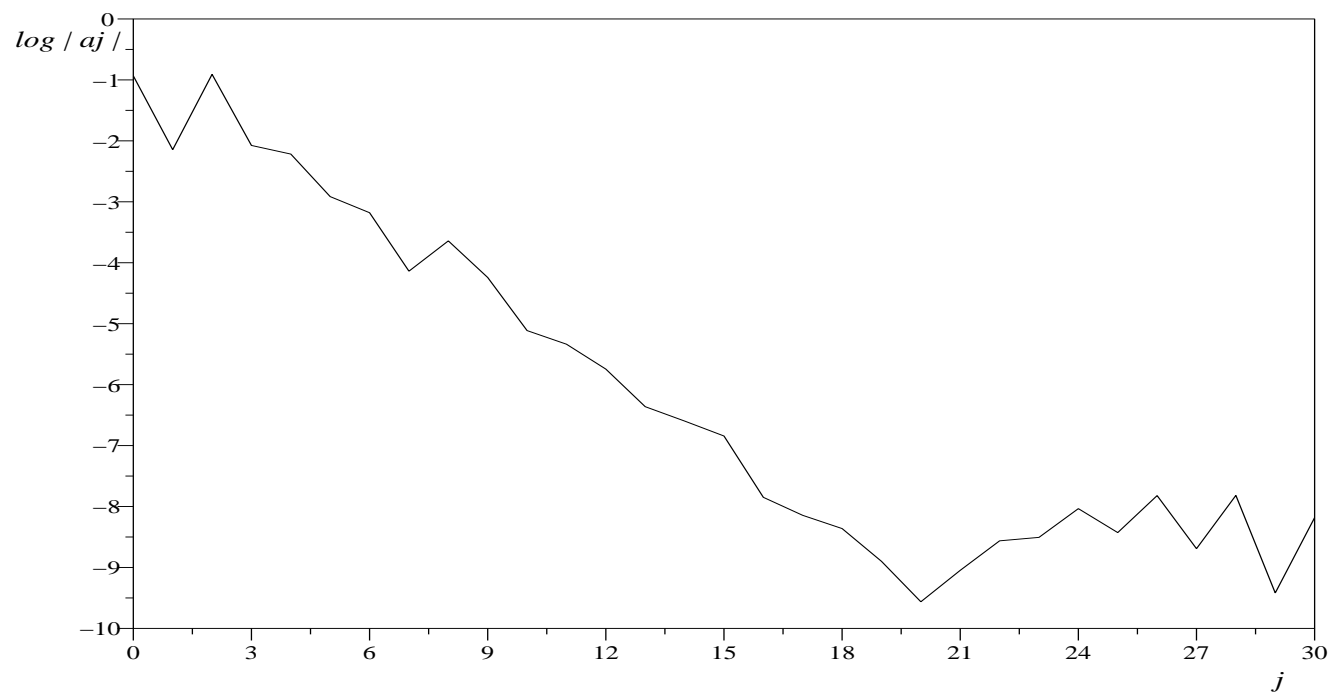

Figure 7 : Spectral convergence for $\mathrm{R}=1.6, \mathrm{Ral}=3000, \mathrm{M}=30$ and $\mathrm{N}=30$ 


\section{Conclusion}

This paper was dedicated to the numerical simulation of an incompressible fluid flow within a cylindrical annular cavity differentially heated. The system is reduced to nonlinear and coupled equations, of Navier-Stokes, energy, and continuity equations.

The natural convection equations, as described, are solved by setting as unknowns : the temperature $T$, the stream function $\Psi$ and the vorticity $\omega$. The numerical simulation by the Collocation-Chebyshev method, allowed us to calculate these variables within the annular layer.

The present numerical code, was improved with the use of diagonalized operators, in connection with the Crank-Nicholson semi-implicit temporal scheme. The results obtained for large ranges of the parameters (Rayleigh number and radius ratio), show the efficiency of this method.

\section{Bibliographie}

[1] C. Bernardi, Y. MADAY, « Approximations spectrales de problèmes aux limites elliptiques », Springer-Verlag France, Paris, 1992.

[2] C. Canuto, M. Y. Hussaini, A. Quarteroni, T. ZAnG, « Spectral Methods in Fluid Dynamics », Springer-Verlag, New York, 1986.

[3] A. Cheddadi, J. P. Caltagirone, A. Mojtabi, K. Vafai, « Free Two-Dimensional Convective Bifurcation in a Horizontal Annulus », ASME J. Heat Transfer, Vol 114, 99-106, 1992.

[4] A. Cheddadi, M. C. Charrier-Mojtabi, A. Mojtabi, «Étude comparative de la convection naturelle dans des espaces annulaires cylindriques fluide et poreux : 1-Ecoulements bidimensionnels multicellulaires », $6^{\text {eme }}$ Colloque Maghrébin sur les Modèles Numériques de l'Ingénieur (C2MNI6), Tunis, 24 - 26 Novembre 1998.

[5] B. Fornberg, «A practical guide to pseudo spectral methods », Cambridge U. Press, 1996.

[6] D. D. Gray, A. Giorgini, « The Validity of the Boussinesq Approximation for Liquids and Gases », International Journal of Heat Mass Transfer. Vol 19, 545-551, 1976.

[7] D. B Haidvogel, T. ZANG, « The accurate solution of Poisson's equation by expansion in Chebyshev polynomials », J. Computational Physics. Vol 30, 167-180, 1979.

[8] T. H. Kuehn, R. J. Goldstein, « An Experimental and Theoretical Study of Natural Convection in the Annulus Between Horizontal Concentric Cylinders », Journal of Fluid Mechanics, Vol 74, 695-719, 1976.

[9] T. H. Kuehn, R. J. Goldstein, « A Parametric Study of Prandtl Number and Diameter Ratio Effects on Natural Convection Heat Transfer in Horizontal Cylindrical Annuli », ASME J. Heat Transfer, Vol 102, 768-770, 1980.

[10] R. PEYRET, «Introduction to Spectral Methods », Von Karman Institute Lecture Series 198604, Rhode-Saint Genese, 1986. 